\title{
FIP1L1-PDGFRalpha Fusion Protein Expression
}

National Cancer Institute

\section{Source}

National Cancer Institute. FIP1L1-PDGFRalpha Fusion Protein Expression. NCI Thesaurus. Code C37217.

Expression of a fusion protein involving the human genes FIP1L1 and PDGFRA. It results from an inversion or translocation and is associated with hypereosinophilic syndrome (HES)/chronic eosinophilic leukemia (CEL) and systemic mast cell disease. 